\title{
The Microbiome and its Impact on Disease in the Preterm Patient
}

\author{
Josef Neu
}

Published online: 1 October 2013

(C) Springer Science + Business Media New York 2013

\begin{abstract}
Emerging technologies derived largely from the Human Genome Project are applied to evaluating the intestinal microbiota in preterm infants. The microbial ecology of the developing intestine is highly related to health and disease, and new discoveries are emerging that will help us understand disorders in the development of the intestinal microbial ecosystem and how to eventually manipulate them to prevent diseases such as necrotizing enterocolitis and late onset sepsis. Here, a brief overview of the developing microbiome as it pertains to several aspects of health and disease in the preterm infant is presented.
\end{abstract}

Keywords Microbiome $\cdot$ Neonate $\cdot$ Intestine $\cdot$ Premature

\section{Introduction}

The intestinal microbiota, a complex ecosystem shaped by the millennia of evolution, normally exists in a commensal and/or symbiotic relationship with the host $[1,2]$. This relationship promotes a delicate equilibrium with the capability to modulate immune responses and promote health. In the past few years, emerging technologies derived largely from the Human Genome Project are applied to evaluating the intestinal microbiota, and new discoveries using these techniques have prompted new initiatives such as the Human Microbiome Roadmap designed to evaluate the role of the intestinal microbiome in health and disease [3].

\section{J. Neu $(\square)$}

Division of Neonatology, Department of Pediatric, University of Florida College of Medicine, 1600 SW Archer Road, Human Development Building HD 112, Gainesville, FL 32610, USA e-mail: neuj@peds.ufl.edu
Our current state of knowledge suggests that the neonatal intestinal microbiota is highly variable in its composition and depends on factors such as gestational age, mode of delivery, type of feeding and other environmental exposures [4]. The host's need for intestinal microbiota is demonstrated by studies showing that germ-free animals do not develop normal lymph node architecture, with extensive defects in the development of gut-associated lymphoid tissue, arrested capillary network development in the gut and reduced antibody production [5].

The traditional view of the human is that it is composed of ten trillion cells, which are the product of $\sim 23,000$ genes. However, it has long been known that in various niches of the human body, there resides a number of species of microbes and microbial genes that vastly outnumber the cells and genes of the human "host".

Until the beginning of the last decade, culture-based techniques were the mainstay of evaluating intestinal microbes. However, it has long been recognized that the majority of bacterial cells seen microscopically in feces are not currently cultured in the laboratory. Recently developed high throughput molecular techniques analyze microbial DNA and RNA and have moved beyond culture-based technologies. There are two general approaches, both of which comprise several variants. One commonly used general approach is to utilize the 16S rRNA gene, and the other is a more complex metagenomic approach in which community DNA is subject to shotgun (whole genome) sequencing. A detailed description of these techniques is beyond the scope of this review and can be found elsewhere $[6 \bullet, 7,8]$.

\section{Adjunctive "Omics" Technologies}

Even if the taxonomy of a particular microbial community is identified, the functional expression as it relates to 
physiology and interaction with the host is not clarified. Simple identification of individual microbes or microbial genotypes often does not clarify their phenotypic expression. Thus, in addition to microbiome identification based technologies, it is important to clarify the function of the microbial communities of interest within a particular niche. It, thus, is of interest to identify the mRNA and protein expression of the genes as well as the metabolites that result before and after interaction of the microbial gene products with the host. Additional "omic" disciplines are, thus, being applied to augment studies of the microbiome. Metatranscriptomics, metaproteinomics, and metabolomics identify gene expression products (mRNA), proteins and metabolites resulting from the genes within a complex microbial community, such as that found in a fecal sample.

Metaproteomics is the study of proteins collectively expressed within microbial communities that usually employ mass spectrometry based analyses to detect proteins associated with the microbiota identified within a given niche. These studies usually also employ a systems biology approach to evaluate the likely functions of the proteins produced by the microbiota. A recent study employed a nontargeted, shotgun mass spectrometry-based whole community proteomics, or metaproteomics, approach for the first deep proteome measurements of thousands of proteins in human fecal samples [9]. The resulting metaproteomes had a distribution that was unexpected relative to the metagenome, with more proteins for translation, energy production and carbohydrate metabolism when compared to what was earlier predicted from metagenomics.

The intestinal microbiota is involved in the regulation of multiple host metabolic pathways, giving rise to interactive host-microbiota metabolic, signaling, and immune-inflammatory processes connecting the intestine, liver, muscle, and brain.

These interactions begin at birth and likely even during fetal life. The microbiota shapes the development of the immune system, and the immune system in turn shapes the composition of the microbiota through a cross talk between the microbes and the host immune system. The signaling processes, together with direct chemical interactions between the microbe and host, act upon multiple organs such as the gut, liver, muscle, and brain.

\section{Intestinal Microecology of the Fetus and Newborn}

Although a commonly held belief is that the intestinal tract of the fetus is sterile, recent studies using a combination of culture and non-culture-based techniques suggest that many preterm infants are exposed to microbes found in the amniotic fluid, even without a history of rupture of membranes or culture-positive chorioamnionitis $[10 \bullet, 11]$. This has led to speculation that the microbial ecology of the swallowed amniotic fluid may have a role not only in the fetal intestinal physiology and inflammation but also perhaps in premature labor [10॰]. Studies of microbial ecology in premature infants using non-culture-based techniques remain few, but this is an area of active investigation.

Recent polymerase chain reaction (PCR)-based studies estimate the prevalence of microbial invasion of the amniotic cavity to be $\geq 30-50 \%$ higher than that detected by cultivation-based methods [12]. From these studies we now know that cultivation-resistant anaerobes belonging to the family Fusobacteriaceae (particularly Sneathia sanguinegens, and Leptotrichia spp.) are also commonly found in amniotic fluid. Other diverse microbes detected by PCR of amniotic fluid include as yet uncultivated and uncharacterized species. A causal relationship between diverse microbes, as detected by PCR, and preterm birth is supported by types of association (e.g., space, time, and dose) proposed as alternatives to Koch's postulates for inferring causality from molecular findings. Whether this colonization affects the gastrointestinal tract of the fetus is not known. We do know, however, that the fetus is known to swallow large quantities of amniotic fluid during the last trimester of pregnancy, and there have been recent findings that microbial DNA is present in meconium. These both suggest that the fetal intestine is exposed to the amniotic fluid microbes. The high sensitivity of the fetal intestine to inflammatory mediators such as LPS $[13,14]$ suggests that microbes may trigger intestinal inflammation in utero. Furthermore, one can speculate that exposure of the intestinal tract to toll like receptor agonists such as LPS, flagellin or microbial $\mathrm{CpG}$ in low quantities may also "tolerize" the intestine to further inflammatory stimuli [15•].

One study used high-throughput $16 \mathrm{~S}$-based techniques to analyze intestinal microbial ecology in premature neonates in 23 neonates born at 23-32 weeks' gestational age [16]. Surprisingly, microbial DNA was detected in meconium, suggesting an intrauterine origin. Differences in diversity were detected in infants whose mothers intended to breast feed (but had not yet fed their babies, suggesting other factors such as socioeconomic or educational status to be important), babies born to mothers treated with antibiotics prior to delivery and in babies born at $<30$ weeks' gestation.

\section{Factors Affecting the Intestinal Microbiota: Postnatal}

Postnatally, many factors can influence intestinal bacterial colonization as well as the responses to colonization. Some studies have shown that the mode of delivery, vaginal versus C-section, type of milk (human milk vs. formula), and antibiotics influence the intestinal flora [17-19]. Those 
infants born via C-section, fed formula milk and exposed to antibiotics have a decrease in diversity of intestinal microbiota and abnormal patterns of colonization with suppression of "healthy" bacteria such as Lactobacillus and Bifidobacteria.

\section{C-Section versus Vaginal Delivery}

Epidemiologic data showing a relationship between increased C-section rate and risk for development of subsequent diseases was recently reviewed [20]. "In the United States the rate of cesarean delivery (CD) has risen $48 \%$ since 1996, reaching a level of $31.8 \%$ in 2007. This trend is reflected in many parts of the world, with the most populous country in the world, China, approaching $50 \%$ and some private clinics in Brazil approaching $80 \%$." In association with the trend of increasing $\mathrm{C}$-section deliveries, there has been an epidemic of both autoimmune diseases such as type 1 diabetes, Crohn's disease, multiple sclerosis, and allergic diseases, such as asthma, allergic rhinitis, and atopic dermatitis. The occurrence of these diseases is higher in more affluent, Western, industrialized countries. As reviewed [20], several previous studies have demonstrated differences in microbial colonization after $\mathrm{C}$-section and vaginal deliveries. A more recent study by Domingiuez-Bello et al. [21], where non-culture-based sequencing technology was used, offers a detailed look at the early stages of the body's colonization by microbes.

During birth and rapidly thereafter, bacteria from the mother and from the surrounding environment colonize the infant's gut. During vaginal delivery, the contact with the mother's vaginal and intestinal flora is an important source of the infant's colonization with a predominance of Lactobacillus, Prevotella and other Bifidobacterium [22, 23] During cesarean delivery, direct contact of the mouth of the newborn with vaginal and intestinal microbiota is absent, and nonmaternally derived environmental bacteria have a more important role in the infants' intestinal colonization, which has a less diverse flora and a bacterial community similar to those found on the skin surface dominated by Staphylococcus and with a delayed intestinal colonization by Lactobacillus, Bifidobacterium and Prevoltella [22, 23].

Some authors have speculated that the composition of the very first human microbiota could have lasting effects on the intestine [20]. Gronlund et al. [24] showed that the primary gut flora in infants born by cesarean delivery may be disturbed for up to 6 months after birth. Another study using culture-based techniques showed that the mode of delivery was associated with differences in intestinal microbes seven years after delivery [25].

There is accumulating evidence that intestinal microbiota has an important role in the postnatal development of the immune system [26]. Available epidemiological data show that atopic diseases, asthma, type 1 diabetes and food allergies appear more often in infants after cesarean delivery than after vaginal delivery [27-30]. Studies in adults using nonculture-based analysis of the intestinal microbiota show that antibiotics may perturb the gastrointestinal tract for years. [31] Since many mothers delivered by $\mathrm{C}$-section also receive antibiotics, whether they have a role in perturbing the newborn intestinal microecology remains unknown but is a confounding factor when one evaluates the epidemiology studies showing atopic disease, type 1 diabetes and food allergies in $\mathrm{C}$-section versus vaginally delivered infants. The composition of enteric microbiota in the early days of life may be a very important factor for achieving and maintaining good health in the years to come. It follows that it is fundamental to identify more thoroughly the intestinal ecosystem during the early developmental stages of life.

\section{Human Milk Versus Infant Formula}

Studies in animals and premature infants have shown that human milk decreases the incidence of necrotizing enterocolitis (NEC) [32, 33]. Beneficial factors of breast milk include immunoglobulins, cytokines, lactoferrin, lysozyme, growth factors, and oligosaccharides (HMO) [17, 34]. Human milk oligosaccharides (HMOs) contain a lactose core and act as prebiotics, stimulating growth of Bifidobacterium species $[35,36]$. At birth a rapid colonization of intestinal flora occurs in the newborn, with aerobic or facultative anaerobic bacteria such as enterobacteria, enterococci and staphylococci represented. These consume oxygen during growth, allowing the proliferation of anaerobic bacteria such as Bacteroides, Bifidobacterias and Clostridia [37, 38]. In the formula-fed infant this transition does not occur, and the newborn intestinal flora differs in its pattern of colonization, with a predominance of Gram negative bacteria and fewer anaerobes [39, 40]. If grouped by phyla, breast-fed infants have a predominance of 'healthy' Firmicutes, mainly Lactobacillus, Bacteroides and Actinobacteria (Bifidobacterium). Formula-fed infants have a predominance of Proteobacteria such as Escherichia coli, and Firmicutes, some of which have pathogenic characteristics such as Clostridia and Staphylococcus. In one study of preterm infants it was speculated that the abnormal pattern of colonization at birth coupled with a delayed bloom of more pathogenic bacteria could potentially lead to the development of NEC [41]. Sullivan et al. [32] in one multicenter study utilizing donor human milk showed that an exclusively human milk-based diet (including human milk based fortifier) is associated with significantly lower rates of NEC, including surgical NEC, when compared with a mother's milk-based diet that also includes bovine milk-based products. The reduction in NEC utilizing strategies of only human milk was $50 \%$ for medical NEC and almost $90 \%$ for surgical NEC. 
The number needed to treat to prevent one case of NEC was estimated to be ten. In a recent AAP policy statement regarding breastfeeding and the use of human milk, the recommendation was to offer donor milk to all preterm infants in which mother's own milk is unavailable [42].

\section{Antibiotic Exposure}

The use of antibiotics is widespread in the intensive care units (NICU), most commonly for a diagnosis of "rule out" infection. Often, their use is justified, but other times it's the product of fear of infections on the part of the care providers. Nevertheless, the use of antibiotics in neonates and especially in the preterm infant has unintended and sometimes adverse consequences. Antibiotic exposure may reduce the diversity of intestinal microbiota, delay the colonization of beneficial bacteria and potentially predispose preterm neonates to NEC. One recent retrospective study found that the duration of antibiotic exposure is associated with an increased risk of NEC among neonates without prior sepsis [43•]. Indeed, studies have shown that overgrowth of pathogenic species is increased after three days of antimicrobial exposure [44]. In the U.S. most mothers giving birth prematurely are treated with antibiotics and most very low birth weight infants are treated with a course of broad spectrum antibiotics such as ampicillin and gentamicin. Studies have shown the detrimental effects of antibiotics to the intestinal flora, even after one dose of antibiotics with alterations in the intestinal microbiota that could take years to recover [18]. One study showed a decrease in diversity, a predominance of less desirable bacteria and highly resistant clones with abundance of specific resistance genes as a result of antibiotic exposure that did not recover after two years post exposure [45]. Cotten et al. [46] found that each empirical treatment day with antibiotic was associated with increased odds of death, necrotizing enterocolitis, and the composite measure of necrotizing enterocolitis or death. They concluded that prolonged initial empirical antibiotic therapy may be associated with increased risk of necrotizing enterocolitis or death and should be used with caution.

Caution should be exercised when deciding to start antibiotics in preterm infants. There are long lasting consequences and life threatening morbidities associated with the indiscriminate use of antibiotics.

\section{Necrotizing Enterocolitis}

Necrotizing Enterocolitis (NEC) is an enigmatic disease that has been recognized for over a century. With the advent of neonatal intensive care, it has become one of the most common and devastating diseases in neonates [47-49]. Complicating the literature is the fact that the causes are multifactorial, and it is not a single disease [48]. For example, when an infant born at term with a hypoplastic left ventricle presents with pneumatosis intestinalis at two days of age, the etiology and pathophysiology of this baby's "NEC" is likely different than the 25 week gestation preterm who presents with pneumatosis intestinalis at five weeks of age. The first is more likely related to ischemic injury due to hemodynamic insufficiency and hypoxicischemic injury [50] rather than a coalescence of factors that result in intestinal inflammation and injury related to intestinal immaturity, as in the preterm infant. In the latter case, sometimes referred to as "classic" NEC [48], the interactions of a predisposing genetic background, an immature intestinal barrier and a microbial environment that is conducive to the development of NEC are thought to play an interactive and critical role in pathogenesis [48, 49, 51].

The linkage of NEC to bacterial colonization was recognized by Santulli et al. [52] over three decades ago. Additional observations showing clusters of cases, outbreaks in institutions, the finding of pneumatosis intestinalis, which likely represents submucosal gas produced by bacterial fermentation, and the common findings of bacteremia and endotoxinemia in affected neonates, supports a microbial role in the pathogenesis of this disease [51]. Numerous bacteria have been related to NEC, but none of them have been found to fulfill Koch's postulates because they are commonly found among patients without NEC [53]. Viruses have also been implicated in the pathogenesis of NEC and Coronavirus within fecal samples and resected intestinal segments were reported in patients with NEC [54], but their actual roles in the causation of the disease have not been substantiated.

\section{Alterations of the Intestinal Microbiota in NEC}

The intestinal microbiota normally exists in a commensal and/or symbiotic relationship with the host [55], but in the neonate and especially in the premature infant, this relationship is underdeveloped and many factors will define its delicate equilibrium with the capability to modulate immune responses and promote health (Fig. 3) [56]. As previously mentioned, a specific pathogen that fulfills Koch's postulates for the etiology of NEC has not been found. Whether the use of a new generation of sequencing technologies will help in this search is unknown, although some preliminary studies are offering clues.

In a study by Wang et al. [57], fecal samples from 20 preterm infants, ten with NEC and ten matched controls (including four twin pairs), were obtained from patients in a single site level III neonatal intensive care unit. Bacterial DNA was subjected to terminal restriction fragment length polymorphism analysis and library sequencing of the $16 \mathrm{~S}$ rRNA gene. The distribution of samples from NEC patients was distinctly different from that of controls, though 
intestinal bacterial colonization in all of these preterm infants was notable for low diversity. Patients with NEC had previously received a higher mean number of days of antibiotics and had even less diversity with an increase in abundance of Gammaproteobacteria and a decrease in other bacteria species. These results suggest a relationship with prior use of antibiotics in patients with NEC, though in this study infants may have been on antibiotics with NEC when the cultures were collected.

In another study by Mai et al. [41], stool specimens were collected prospectively from infants with gestational ages $\leq 32$ completed weeks or birth weights $\leq 1250 \mathrm{~g}$, starting with the first stool and continuing weekly until discharge. High throughput 16S rRNA sequencing was used to compare the diversity of microbiota and the prevalence of specific bacterial signatures in nine NEC infants and in nine matched controls. A bloom (34\% increase) of Proteobacteria and a decrease $(32 \%)$ in Firmicutes in NEC cases between the 1 week and $<72 \mathrm{~h}$ was detected. No significant change was identified in the controls. Several molecular signatures were increased in the NEC cases one week before and within $72 \mathrm{~h}$ of NEC development. One of the bacterial signatures detected more frequently in NEC cases $(p<0.01)$ matched closest to $\gamma$-Proteobacteria, also noted in the Wang study [57]. Although this sequence grouped with the well studied Enterobacteriaceae family, it did not match any sequence in Genbank by more than $97 \%$, suggesting that novel pathogens contribute to the etiology of NEC.

\section{Conclusion}

The current evidence highlights the importance of the development of a healthy intestinal microbiota in the neonate. Many aspects that promote health or disease are related to the homeostasis of this intestinal microbiota. Especially in the preterm infant, the delicate equilibrium of the intestinal microbiota could be strongly influenced by the intervention that we as physicians routinely perform as part of the medical care of the neonate. As the awareness of the importance of the development and maintenance of the intestinal flora increases and the development of newer molecular techniques allows us to identify, monitor and modify this microbiota we will be able to improve the care of our infants with interventions with long lasting effects.

\section{Compliance with Ethics Guidelines}

Conflict of Interest Josef Neu is on the Scientific Advisory Board for Medela and Mead Johnson, has consulted for Biogaia, was a mentor to a postdoctoral fellow who received a research grant from Gerber and has been supported to travel to meetings by Nestle, Danone and Abbott.
Human and Animal Rights and Informed Consent This article does not contain any studies with human or animal subjects performed by any of theauthors

\section{References}

Recently published papers of particular interest have been highlighted as:

- Of importance

1. Hooper LV. Bacterial contributions to mammalian gut development. Trends Microbiol. 2004;12:129-34.

2. Hooper LV, Gordon JI. Commensal host-bacterial relationships in the gut. Science. 2001;292:1115-8.

3. Turnbaugh PJ, Ley RE, Hamady M, Fraser-Liggett CM, Knight R, Gordon JI. The human microbiome project. Nature. 2007;449: 804-10.

4. Penders J, Thijs C, Vink C, et al. Factors influencing the composition of the intestinal microbiota in early infancy. Pediatrics. 2006;118:511-2.

5. Stappenbeck TS, Hooper LV, Gordon JI. Developmental regulation of intestinal angiogenesis by indigenous microbes via Paneth cells. Proc Natl Acad Sci USA. 2002;99:15451-5.

6. - Hattori M, Taylor TD. The human intestinal microbiome: a new frontier of human biology. DNA Res. 2009;16:1-12. This review discusses the advantages over culture based techniques and challenges of studying microbes using next-generation sequencing technologies. It provides an overview and comparison of techniques such as $16 \mathrm{~S}$ sequencing versus shotgun and some of the bioinformatics software available to analyze data generated by these technologies.

7. Hamady M, Knight R. Microbial community profiling for human microbiome projects: tools, techniques, and challenges. Genome Res. 2009;19:1141-52.

8. Weinstock GM. Genomic approaches to studying the human microbiota. Nature. 2012;489:250-6.

9. Verberkmoes NC, Russell AL, Shah M, et al. Shotgun metaproteomics of the human distal gut microbiota. ISME J. 2009;3:179-89.

10. - DiGiulio DB. Diversity of microbes in amniotic fluid. Semin Fetal Neonatal Med. 2012;17:2-11. Discusses various microbes found in association with microbial invasion of amniotic fluid without rupture of membranes and raises numerous questions of whether these microbes may be responsible for preterm labor and postnatal diseases where early colonization may have an effect on development of the immune system.

11. DiGiulio DB, Romero R, Amogan HP, et al. Microbial prevalence, diversity and abundance in amniotic fluid during preterm labor: a molecular and culture-based investigation. PLoS ONE. 2008;3:33056.

12. DiGiulio DB, Gervasi MT, Romero R, et al. Microbial invasion of the amniotic cavity in pregnancies with small-for-gestationalage fetuses. J Perinat Med. 2010;38:495-502.

13. Nanthakumar N, Meng D, Goldstein AM, et al. The mechanism of excessive intestinal inflammation in necrotizing enterocolitis: an immature innate immune response. PLoS One. 2011;6:e17776.

14. Nanthakumar NN, Fusunyan RD, Sanderson I, Walker WA. Inflammation in the developing human intestine: a possible pathophysiologic contribution to necrotizing enterocolitis. Proc Natl Acad Sci USA. 2000;97:6043-8.

15. - Medzhitov R, Schneider DS, Soares MP. Disease tolerance as a defense strategy. Science. 2012;335:936-41. Discusses how the 
immune system protects from infections primarily by detecting and eliminating the invading pathogen but also how the host organism can protect itself via the mechanisms of tolerance. This is a "distinct host defense strategy, which has been largely overlooked in animal and human studies," that may provide new approaches for the treatment of infections and other diseases.

16. Mshvildadze M, Neu J, Schuster J, Theriaque D, Li N, Mai V. Intestinal microbial ecology in premature infants assessed with non-culture-based techniques. J Pediatr. 2010;156:20-5.

17. Harmsen HJ, Wildeboer-Veloo AC, Raangs GC, et al. Analysis of intestinal flora development in breast-fed and formula-fed infants by using molecular identification and detection methods. J Pediatr Gastroenterol Nutr. 2000;30:61-7.

18. Jernberg C, Lofmark S, Edlund C, Jansson JK. Long-term ecological impacts of antibiotic administration on the human intestinal microbiota. ISME J. 2007;1:56-66.

19. Gronlund MM, Lehtonen OP, Eerola E, Kero P. Fecal microflora in healthy infants born by different methods of delivery: permanent changes in intestinal flora after cesarean delivery. J Pediatr Gastroenterol Nutr. 1999;28:19-25.

20. Neu J, Rushing J. Cesarean versus vaginal delivery: long-term infant outcomes and the hygiene hypothesis. Clin Perinatol. 2011;38:321-31.

21. Dominguez-Bello MG, Blaser MJ, Ley RE, Knight R. Development of the human gastrointestinal microbiota and insights from high-throughput sequencing. Gastroenterology. 2011;140: 1713-9.

22. Dominguez-Bello MG, Costello EK, Contreras M, et al. Delivery mode shapes the acquisition and structure of the initial microbiota across multiple body habitats in newborns. Proc Natl Acad Sci USA. 2010;107:11971-5.

23. Biasucci G, Benenati B, Morelli L, Bessi E, Boehm G. Cesarean delivery may affect the early biodiversity of intestinal bacteria. J Nutr. 2008;138:1796S-800S.

24. Grönlund MM, Lehtonen OP, Eerola E, Kero P. Fecal microflora in healthy infants born by different methods of delivery: permanent changes in intestinal flora after cesarean delivery. J Pediatr Gastroenterol Nutr. 1999;28:19-25.

25. Salminen S, Gibson GR, McCartney AL, Isolauri E. Influence of mode of delivery on gut microbiota composition in seven year old children. Gut. 2004;53:1388-9.

26. Björkstén B. Effects of intestinal microflora and the environment on the development of asthma and allergy. Springer Semin Immunopathol. 2004;25:257-70.

27. Negele K, Heinrich J, Borte M, et al. Mode of delivery and development of atopic disease during the first 2 years of life. Pediatr Allergy Immunol. 2004;15:48-54.

28. Debley JS, Smith JM, Redding GJ, Critchlow CW. Childhood asthma hospitalization risk after cesarean delivery in former term and premature infants. Ann Allergy Asthma Immunol. 2005;94:228-33.

29. Laubereau B, Filipiak-Pittroff B, von Berg A, et al. Caesarean section and gastrointestinal symptoms, atopic dermatitis, and sensitisation during the first year of life. Arch Dis Child. 2004;89:993-7.

30. Eggesbø M, Botten G, Stigum H, Nafstad P, Magnus P. Is delivery by cesarean section a risk factor for food allergy? J Allergy Clin Immunol. 2003;112:420-6.

31. Jernberg C, Löfmark S, Edlund C, Jansson JK. Long-term ecological impacts of antibiotic administration on the human intestinal microbiota. ISME J. 2007;1:56-66.

32. Sullivan S, Schanler RJ, Kim JH, et al. An exclusively human milk-based diet is associated with a lower rate of necrotizing enterocolitis than a diet of human milk and bovine milk-based products. J Pediatr. 2010;156:562-7.
33. Barlow B, Santulli TV, Heird WC, Pitt J, Blanc WA, Schullinger JN. An experimental study of acute neonatal enterocolitis-the importance of breast milk. J Pediatr Surg. 1974;9:587-95.

34. Hanson LA, Korotkova M, Telemo E. Breast-feeding, infant formulas, and the immune system. Ann Allergy Asthma Immunol. 2003;90:59-63.

35. Ward RE, Ninonuevo M, Mills DA, Lebrilla CB, German JB. In vitro fermentation of breast milk oligosaccharides by Bifidobacterium infantis and Lactobacillus gasseri. Appl Environ Microbiol. 2006;72:4497-9.

36. Ninonuevo MR, Park Y, Yin H, et al. A strategy for annotating the human milk glycome. J Agric Food Chem. 2006;54:7471-80.

37. Adlerberth I, Lindberg E, Aberg N, et al. Reduced enterobacterial and increased staphylococcal colonization of the infantile bowel: an effect of hygienic lifestyle? Pediatr Res. 2006;59:96-101.

38. Bjorkstrom MV, Hall L, Soderlund S, Hakansson EG, Hakansson $\mathrm{S}$, Domellof M. Intestinal flora in very low-birth weight infants. Acta Paediatr. 2009;98:1762-7.

39. Le Huerou-Luron I, Blat S, Boudry G. Breast- v. formula-feeding: impacts on the digestive tract and immediate and long-term health effects. Nutr Res Rev. 2010;23:23-36.

40. Penders J, Thijs C, Vink C, et al. Factors influencing the composition of the intestinal microbiota in early infancy. Pediatrics. 2006;118:511-21.

41. Mai V, Young CM, Ukhanova M, et al. Fecal microbiota in premature infants prior to necrotizing enterocolitis. PLoS One. 2011;6:e20647.

42. Breastfeeding SO. Breastfeeding and the Use of Human Milk. Pediatrics. 2012;129:e827-41.

43. - Alexander VN, Northrup V, Bizzarro MJ. Antibiotic exposure in the newborn intensive care unit and the risk of necrotizing enterocolitis. J Pediatr. 2011;159:392-7. A case control study that shows a link between antibiotic usage and the development of necrotizing enterocolitis. This is an example of how manipulation of of the microbiome may have inadvertent consequences.

44. Goldmann DA, Leclair J, Macone A. Bacterial colonization of neonates admitted to an intensive care environment. J Pediatr. 1978;93:288-93.

45. Jakobsson HE, Jernberg C, Andersson AF, Sjolund-Karlsson M, Jansson JK, Engstrand L. Short-term antibiotic treatment has differing long-term impacts on the human throat and gut microbiome. PLoS One. 2010;5:e9836.

46. Cotten CM, Taylor S, Stoll B, et al. Prolonged duration of initial empirical antibiotic treatment is associated with increased rates of necrotizing enterocolitis and death for extremely low birth weight infants. Pediatrics. 2009;123:58-66.

47. Obladen M. Necrotizing enterocolitis-150 years of fruitless search for the cause. Neonatology. 2009;96:203-10.

48. Neu J, Walker WA. Necrotizing enterocolitis. N Engl J Med. 2011;364:255-64.

49. Lin PW, Stoll BJ. Necrotising enterocolitis. The Lancet. 2006;368:1271-83.

50. Young CM, Kingma SD, Neu J. Ischemia-reperfusion and neonatal intestinal injury. J Pediatr. 2011;158:e25-8.

51. Claud EC, Walker WA. Hypothesis: inappropriate colonization of the premature intestine can cause neonatal necrotizing enterocolitis. FASEB J. 2001;15:1398-403.

52. Sántulli TV, Schullinger JN, Heird WC, et al. Acute necrotizing enterocolitis in infancy: a review of 64 cases. Pediatrics. 1975;55:376-87.

53. Falkow S. Molecular Koch's postulates applied to bacterial pathogenicity-a personal recollection 15 years later. Nat Rev Microbiol. 2004;2:67-72. 
54. Resta S, Luby JP, Rosenfeld CR, Siegel JD. Isolation and propagation of a human enteric coronavirus. Science. 1985;229:978-81.

55. Hooper LV, Gordon JI. Commensal host-bacterial relationships in the gut. Science. 2001;292:1115-8.

56. Forsythe $\mathrm{P}$, Bienenstock J. Immunomodulation by commensal and probiotic bacteria. Immunol Invest. 2010;39:429-48.
57. Wang Y, Hoenig JD, Malin KJ, et al. 16S rRNA gene-based analysis of fecal microbiota from preterm infants with and without necrotizing enterocolitis. ISME J. 2009;3:944-54. 